\title{
Effects of Polarizability on the Hydration of the Chloride Ion
}

\author{
Steven J. Stuart and B. J. Berne* \\ Department of Chemistry and the Center for Biomolecular Simulation, Columbia University, \\ New York, New York 10027
}

Received: April 10, 1996; In Final Form: May 13, $1996^{\otimes}$

\begin{abstract}
Polarizable and nonpolarizable potential models for both water and chloride are used to address the issue of surface $v$ s interior solvation of the chloride ion in $\mathrm{Cl}\left(\mathrm{H}_{2} \mathrm{O}\right)_{n}{ }^{-}$clusters, for $n$ up to 255 . We find that, even for the largest clusters, simulations with polarizable water models show that the chloride ion is preferentially solvated near the surface of the cluster. This behavior is not observed with a nonpolarizable model. The many-body effects are not directly responsible for this solvation behavior; polarizability appears to be important primarily for its role in facilitating a larger average dipole moment on the water model. Polarizability on the chloride ion is not found to have a substantial effect on the structure of the clusters.
\end{abstract}

\section{Introduction}

There has been considerable theoretical and experimental interest in the solvation of ions by small molecular clusters as a model for bulk solvation. ${ }^{1}$ In particular, there has recently been some controversy concerning the structure of aqueous clusters of the chloride ion. ${ }^{2-4}$

Perera and Berkowitz reported that in clusters of a $\mathrm{Cl}^{-}$ion with up to 20 polarizable water molecules the ion remains on the surface of the cluster, asymmetrically solvated by the water molecules. ${ }^{3-6}$ This result was somewhat surprising, as it had been generally assumed that successive water molecules would incrementally fill the first solvation shell before beginning to fill the second. ${ }^{7,8}$ Furthermore, both experiment ${ }^{9}$ and simulation $^{10}$ agree that chloride ions are solvated away from the surface at a flat air-water interface, which is essentially a very large cluster.

Perera and Berkowitz argue that observation of surface solvation depends on the proper treatment of many-body polarization effects and that nonpolarizable models do not display this behavior. ${ }^{3,4}$ Jorgensen and Severance dispute these results, pointing out an error in the original Perera and Berkowitz paper and arguing that polarization should be important only for ions with stronger electric fields. ${ }^{2}$ They also demonstrate that water molecules are distributed asymmetrically about the chloride ion when simulated with their nonpolarizable model. Perera and Berkowitz subsequently corrected the flaws in their initial comparison and still maintain that polarization is necessary to generate structurally correct $\mathrm{Cl}\left(\mathrm{H}_{2} \mathrm{O}\right)_{n}{ }^{-}$clusters. ${ }^{4}$

These provocative studies leave several questions unanswered (or worse, doubly answered). Do nonpolarizable models place the chloride on the inside of the clusters, as claimed by Perera and Berkowitz, or on the outside, as claimed by Jorgensen and Severance? If the solvation behavior is in fact different when polarization is included, what is it about the polarizable models that drives the chloride ion to the outside of the cluster? Presumably the ion will withdraw to the interior as the cluster becomes larger: at what cluster sizes does this occur? And why?

We attempt to answer these questions in this paper. The structures of these clusters are analyzed in detail to determine if there is any substantial difference in the predictions of polarizable and nonpolarizable models. Simulations are per-

${ }^{\otimes}$ Abstract published in Advance ACS Abstracts, July 1, 1996. formed on a sequence of $\mathrm{Cl}\left(\mathrm{H}_{2} \mathrm{O}\right)_{n}{ }^{-}$clusters, up to $n=255$, to observe any trends in solvation behavior as the cluster size approaches infinity. By using several combinations of polarizable and nonpolarizable models, we can begin to understand what features of the models are responsible for driving the chloride ion to the surface of the clusters.

In section 2 we describe the polarizable and nonpolarizable models that will be used. One of these is a new polarizable ion model, which we describe in some detail. Section 3 outlines the extended Lagrangian dynamics algorithm used to simulate the polarizable systems, section 4 describes the simulation conditions, and section 5 reports the results for both bulk and cluster simulations. The significance of the results is discussed in section 6 .

\section{Models}

2.1. Nonpolarizable Water Model. The TIP4P water model (also known as OPLS), ${ }^{11}$ one of the most widely used water models due to its accuracy and relatively low computational cost, is the nonpolarizable water model used here. This is the same model used in the cluster studies of Perera and Berkowitz $^{3,4}$ and Jorgensen and Severance. ${ }^{2}$

In this potential model, the water molecules are constrained to have the experimental gas-phase water geometry $\left(r_{\mathrm{OH}}=\right.$ $\left.0.9572 \AA, \angle \mathrm{HOH}=104.52^{\circ}\right)$. The two hydrogen atoms have fixed point charges of $0.52|e|$, and a single negative charge of $-1.04|e|$ is located $0.15 \AA$ from the oxygen along the $\mathrm{HOH}$ bisector. This results in a molecular dipole moment of $2.18 \mathrm{D}$, which is larger than the gas-phase dipole of $1.85 \mathrm{D},{ }^{12}$ but smaller than the estimated liquid-state value of $2.5-2.6 \mathrm{D} .{ }^{13-16}$

In the TIP4P water model the point charges interact through a $1 / r$ electrostatic interaction, and the oxygen sites interact through a Lennard-Jones (LJ) potential. There are no intramolecular interactions. The potential energy of a system of TIP4P molecules can thus be written

$$
\begin{aligned}
V[\{\mathbf{r}\}]=\frac{1}{2} \sum_{i \alpha} \sum_{j \beta, j \neq i} q_{i \alpha} q_{j \beta} \frac{1}{r_{i \alpha j \beta}}+ & \\
& \frac{1}{2} \sum_{i} \sum_{j \neq i} 4 \epsilon\left[\left(\frac{\sigma}{r_{i \mathrm{O} j \mathrm{O}}}\right)^{12}-\left(\frac{\sigma}{r_{i \mathrm{O} j \mathrm{O}}}\right)^{6}\right]
\end{aligned}
$$

where the subscript $i \alpha$ denotes site $\alpha$ on molecule $i$.

The parameters used in this model are summarized in Table 1. 
TABLE 1: Parameters for the TIP4P and TIP4P-FQ Water Models

\begin{tabular}{|c|c|c|}
\hline & TIP4P $^{a}$ & TIP4P-FQ \\
\hline \multicolumn{3}{|c|}{ Model Parameters } \\
\hline$\epsilon(\mathrm{kcal} / \mathrm{mol})$ & $0.1550^{b}$ & 0.2862 \\
\hline$\sigma(\AA)$ & $3.154^{b}$ & 3.159 \\
\hline$\theta_{\mathrm{HOH}}(\mathrm{deg})$ & 104.52 & 104.52 \\
\hline$r_{\mathrm{OH}}(\AA)$ & 0.9572 & 0.9572 \\
\hline$r_{\mathrm{OM}}(\AA)$ & 0.15 & 0.15 \\
\hline$q_{\mathrm{H}}$ & 0.52 & \\
\hline$\chi_{\mathrm{O}}-\chi_{\mathrm{H}}\left(\mathrm{kcal} \mathrm{mol}^{-1} e^{-1}\right)$ & & 68.49 \\
\hline$\zeta_{0}\left(\AA^{-1}\right)$ & & 3.08 \\
\hline$\xi_{\mathrm{H}}\left(\AA^{-1}\right)$ & & 1.70 \\
\hline \multicolumn{3}{|c|}{ Derived Quantities } \\
\hline$J_{\mathrm{OO}}^{0}\left(\mathrm{kcal} \mathrm{mol}^{-1} e^{-2}\right)$ & & 371.6 \\
\hline$J_{\mathrm{HH}}^{0}\left(\mathrm{kcal} \mathrm{mol}^{-1} e^{-2}\right)$ & & 353.0 \\
\hline$J_{\mathrm{OH}}\left(r_{\mathrm{MH}}\right)\left(\mathrm{kcal} \mathrm{mol}^{-1} e^{-2}\right)$ & & 286.4 \\
\hline$J_{\mathrm{HH}}\left(r_{\mathrm{HH}}\right)\left(\mathrm{kcal} \mathrm{mol}^{-1} e^{-2}\right)$ & & 203.6 \\
\hline
\end{tabular}

${ }^{a}$ Reference $11 .{ }^{b} n . b .:$ Reference 11 defines the TIP4P water model in terms of $A$ and $C$ coefficients instead of $\epsilon$ and $\sigma$. This is equivalent to the values given here for bulk water, but care must be taken to use the appropriate combining rule in mixed systems.

2.2. Polarizable Water Model. For our polarizable water model, we use a fluctuating-charge version of the TIP4P model (TIP4P-FQ). ${ }^{17}$ This is a polarizable model that has proved useful in the study of a variety of aqueous solvation effects ${ }^{18-20}$ and that offers considerable advantages over dipole-polarizable models in computational efficiency. Fluctuating-charge models such as TIP4P-FQ incorporate many-body polarization interactions by allowing the partial charges in the simulation to vary as additional degrees of freedom. These charge degrees of freedom are propagated in the molecular dynamics simulation with an adiabatic extended-Lagrangian algorithm, ${ }^{21,22}$ using very little additional computation. This differs from dipole-polarizable models, such as the SPCE/POL model ${ }^{23,24}$ used in the simulations of Perera and Berkowitz, which use constant partial charges and polarizable point dipoles. These dipole-dipole interactions require an additional term in the potential that is expensive to calculate and must be iterated to a self-consistent solution.

The TIP4P-FQ model is based on the TIP4P model in the sense that it shares the same geometry. The Lennard-Jones parameters are different, however, and there are a few additional interactions.

The potential for a system of $N$ TIP4P-FQ water molecules has the form

$$
\begin{aligned}
& V[\{\mathbf{r}\},\{q\}]=\sum_{i \alpha}\left[\chi_{i \alpha}^{0} q_{i \alpha}+\frac{1}{2} J_{i \alpha i \alpha}^{0} q_{i \alpha}^{2}\right]+ \\
& \frac{1}{2} \sum_{i \alpha} \sum_{j \beta \neq i \alpha} q_{i \alpha} q_{j \beta} J_{i \alpha j \beta}\left(r_{i \alpha j \beta}\right)+\frac{1}{2} \sum_{i} \sum_{j \neq i} 4 \epsilon\left[\left(\frac{\sigma}{r_{i \mathrm{O} j \mathrm{O}}}\right)^{12}-\right. \\
& \\
& \left.\left(\frac{\sigma}{r_{i \mathrm{O} j \mathrm{O}}}\right)^{6}\right]-N V^{\mathrm{gp}}
\end{aligned}
$$

The first term, which is not present in the TIP4P model, describes the variation of a single atom's energy with its partial charge. The coefficients $\chi^{0}$ and $J^{0}$ depend on atom type and may be equated with the atom's electronegativity and hardness via density functional theory. ${ }^{25,26}$ Terms of this type are not required in fixed-charge models, as they remain constant in the absence of variable charges. Their evaluation in FQ models is quite simple, however, and they represent a very small part of the computational effort.
Since a fluctuating-charge model includes intramolecular charge interactions, the traditional $1 / r$ Coulomb interaction is not desirable. Instead, the charges interact as if they were delocalized, using a Coulomb integral of the form

$$
\begin{aligned}
& J_{i \alpha j \beta}\left(r_{i \alpha j \beta}\right)= \\
& \quad \int \mathrm{d} \mathbf{r}_{1} \int \mathrm{d} \mathbf{r}_{2}\left|\psi_{i \alpha}\left(\mathbf{r}_{1}\right)\right|^{2} \frac{1}{\left|\mathbf{r}_{1}-\mathbf{r}_{2}-\left(\mathbf{r}_{i \alpha}-\mathbf{r}_{j \beta}\right)\right|}\left|\psi_{j \beta}\left(\mathbf{r}_{2}\right)\right|^{2}
\end{aligned}
$$

where the $\psi_{i \alpha}$ are single Slater s orbitals

$$
\psi_{i \alpha}(r)=A_{i \alpha} r^{n_{i \alpha}-1} \mathrm{e}^{-\xi_{i \alpha} r}
$$

with appropriate principal quantum number $n$ and normalization constant $A$. The orbital exponent $\zeta$ is an adjustable parameter that controls the extent of the charge delocalization and is also used to specify the value of $J_{i \alpha i \alpha}^{0}$,

$$
J_{i \alpha i \alpha}^{0}=\lim _{r_{i \alpha j \beta} \rightarrow 0} J_{i \alpha j \beta}\left(r_{i \alpha j \beta}\right)
$$

for chemically equivalent atoms $i \alpha$ and $j \beta$. This $J(r)$ interaction differs significantly from $1 / r$ only at distances smaller than 3 $\AA$ for typical values of $\zeta$. Thus for computational convenience the TIP4P-FQ model approximates $J_{\mathrm{OO}}(r), J_{\mathrm{OH}}(r)$, and $J_{\mathrm{HH}}(r)$ by $1 / r$ for all intermolecular interactions; only the intramolecular interactions require the evaluation of a Coulomb integral. There are relatively few of these intramolecular interactions $(O(N)$ as opposed to $0\left(N^{2}\right)$ ), and they occur at fixed bond lengths in the rigid TIP4P-FQ model, so this new interaction also contributes very little to the computational effort.

In contrast to the fixed-charge TIP4P model, the energy of a single gas-phase TIP4P-FQ water molecule is nonzero. Thus this gas-phase energy is subtracted from the potential in eq 2 so that the energy zero corresponds to a gas of molecules at infinite separation. Once again, this new term may be precalculated and requires almost no extra computation.

With a potential so similar to that of conventional fixedcharge force fields, the only significant extra work that must be done is the propagation of an extra $N$ degrees of freedom in the dynamics algorithm, a portion of the algorithm which requires much less time than the calculation of the energies and forces. Consequently, this polarizable model needs only $5-10 \%$ more CPU time than TIP4P. But the inclusion of polarizability results in a model that is considerably more accurate than fixed charge models, particularly for dielectric and dynamic properties. ${ }^{17}$ The accuracy is comparable to that achieved by dipolepolarizable models, which typically require $2-4$ times as much computational effort. ${ }^{27}$

Computational efficiency aside, there are other differences to consider between fluctuating-charge and dipole-polarizable models. In the latter, the many-body interactions are treated explicitly at dipolar order; higher order (dipole-quadrupole, etc.) terms are not included. The fluctuating-charge model, by treating the charge interactions directly, preserves all higher order multipolar interactions. With this model, however, the symmetry of the polarizability tensor may be restricted by the locations of the partial charges. A planar model, such as the TIP4P-FQ water model, will have no out-of-plane polarizability components.

The parameters that define the TIP4P-FQ model are summarized in Table 1.

2.3. Nonpolarizable Chloride Ion Model. The nonpolarizable chloride ion model consists of a unit negative charge that interacts via $1 / r$ and $\mathrm{LJ}$ potentials with other charges and $\mathrm{LJ}$ sites in the system. The version we use here is the OPLS model 
TABLE 2: Parameters for the OPLS and Drude Ion Models for $\mathrm{Cl}^{-}$

\begin{tabular}{|c|c|c|}
\hline & OPLS $^{a}$ & Drude ion \\
\hline \multicolumn{3}{|c|}{ Fundamental Parameters } \\
\hline$M(\mathrm{amu})$ & 35.453 & 35.453 \\
\hline$Q(e)$ & -1.0 & -1.0 \\
\hline \multicolumn{3}{|c|}{ Adjustable Parameters } \\
\hline$\alpha\left(\AA^{3}\right)$ & & 3.76 \\
\hline$\zeta\left(\AA^{-1}\right)$ & & 2.307 \\
\hline$\epsilon(\mathrm{kcal} / \mathrm{mol})$ & $0.1178^{b}$ & 0.0299 \\
\hline$\sigma(\AA)$ & $4.311^{b}$ & 4.00 \\
\hline \multicolumn{3}{|c|}{ Algorithmic Parameters } \\
\hline$q(e)$ & & 5 \\
\hline$\omega\left(\mathrm{fs}^{-1}\right)$ & & 3.45 \\
\hline \multicolumn{3}{|c|}{ Derived Quantities } \\
\hline$\mu(\mathrm{amu})$ & & 0.090 \\
\hline$\delta m=m_{\mathrm{sat}}(\mathrm{a}$ & & 0.090 \\
\hline$M-\delta m=n$ & & 35.363 \\
\hline
\end{tabular}

${ }^{a}$ Reference 28. ${ }^{b} n . b .:$ Reference 28 defines the OPLS $\mathrm{Cl}^{-}$model in terms of $A$ and $C$ coefficients instead of $\epsilon$ and $\sigma$. This is equivalent to the values given here and in Table 1 when a single $\mathrm{Cl}^{-}$ion is interacting with TIP4P water, but care must be taken to use the appropriate combining rule when more than one ion is present.

developed by Jorgensen et al., ${ }^{28}$ which was also used in the cluster studies of Perera and Berkowitz ${ }^{3,4}$ and Jorgensen and Severance. $^{2}$ This model has been specifically parametrized against the TIP4P water model and produces reasonable pair correlation functions in dilute solution. The coordination number is known to be too large, however, as with many fixedcharge ion models. The parameters for the OPLS chloride model are summarized in Table 2 .

2.4. Polarizable Chloride Ion Model. The polarizable chloride ion that we use here is a new one, developed by us specifically for use with the TIP4P-FQ water model. ${ }^{29}$ It is related to both the Drude dispersion oscillator ${ }^{30-32}$ and the shell model, ${ }^{33}$ both of which have also been used to simulate polarizable atomic fluids.

A fluctuating-charge model of the usual type is not possible for a monatomic ion, due to its single charge site: if the charge of the ion is constrained at $-1|e|$, there are no other sites to which charge may be transferred. Even if intermolecular charge transfer were permitted (and it is not observed ${ }^{1}$ ), there would be no way to induce a purely ionic dipole moment. Instead, we use a modified Drude dispersion oscillator to model the induced dipole. In contrast to traditional uses of the Drude oscillator, however, we simulate the spring at very low temperatures to ensure that it maintains the correct dipole. We make use of the Drude model only for its polarizability and do not rely on its fluctuation properties, which would give rise to the classical London dispersion force.

The Drude ion model used here consists of two partial charges, $q$ and $Q-q$, connected by a three-dimensional harmonic spring of frequency $\omega$. The ion has a net charge of $Q=-1|e|$, appropriate for the chloride ion. The charge $q$ has a mass of $\delta m$ and the charge $Q-q$ has a charge of $M-\delta m$, for a chloride ion mass of $M=35.453$ amu. As implied by the notation, we will choose $\delta m \ll M$ so that the heavier site remains near the center of mass as the spring oscillates.

The two charges do not interact with each other Coulombically: their only interaction is the harmonic potential in the spring displacement $\mathbf{d}=\mathbf{R}-\mathbf{r}$, where $\mathbf{R}$ and $\mathbf{r}$ are the positions of the heavy and light charges, respectively. In the presence of a constant external electric field $\mathbf{E}$, then, the Drude ion feels a potential

$$
V(\mathbf{d}, \mathbf{R})=\frac{1}{2} \mu \omega^{2} d^{2}-q \mathbf{d} \cdot \mathbf{E}-Q \mathbf{R} \cdot \mathbf{E}
$$

where

$$
\mu=\delta m\left(\frac{M-\delta m}{M}\right)
$$

is the reduced mass of the oscillator. This potential will be minimized by taking

$$
\mathbf{d}=\frac{q}{\mu \omega^{2}} \mathbf{E}
$$

for an ionic dipole $\mathbf{p}$ of

$$
\mathbf{p}=\frac{q^{2}}{\mu \omega^{2}} \mathbf{E}
$$

demonstrating that the polarizability of the Drude oscillator is

$$
\alpha=\frac{q^{2}}{\mu \omega^{2}}
$$

As with the OPLS potential, the interactions between chloride ions and water molecules include both electrostatic and LJ potentials. The electrostatic interactions are handled using a $J(r)$ interaction between delocalized charges, as for the TIP4PFQ model. Thus the potential for a mixed system of TIP4PFQ water molecules and Drude ions has the form

$$
\begin{array}{r}
V[\{\mathbf{r}\},\{q\}]=\sum_{i \in \text { Drude }} \frac{1}{2} \mu_{i} \omega_{i}^{2} d_{i}^{2}+\sum_{i \alpha \in \mathrm{FQ}}\left[\chi_{i \alpha}^{0} q_{i \alpha}+\frac{1}{2} J_{i \alpha i \alpha}^{0} q_{i \alpha}^{2}\right]+ \\
\frac{1}{2} \sum_{i \alpha} \sum_{j \beta \neq i \alpha}{ }^{\prime} q_{i \alpha} q_{j \beta} J_{i \alpha j \beta}\left(r_{i \alpha j \beta}\right)+\frac{1}{2} \sum_{i \alpha \in \mathrm{LJ}} \sum_{\substack{j \beta \in \mathrm{LJ} \\
i \neq j}} 4 \epsilon_{i \alpha j \beta}\left[\left(\frac{\sigma_{i j}}{r_{i j}}\right)^{12}-\right. \\
\left.\left(\frac{\sigma_{i j}}{r_{i j}}\right)^{6}\right]-\sum_{i} V_{i}^{\mathrm{gp}}(11)
\end{array}
$$

Note that the Drude displacement variables $\mathbf{d}_{i}$ may be expressed in terms of the charge positions $\mathbf{r}_{i \alpha}$. The two charges on each Drude ion are delocalized, as in the TIP4P-FQ model, and thus require a $\zeta$ parameter for the $J(r)$ interaction. These are not FQ charges, however, and do not have $\chi^{0}$ or $J^{0}$ parameters. There is no intraionic Coulomb interaction; the prime on the electrostatic sum indicates that the intramolecular Drude ion interactions should be skipped. We use the full $J(r)$ potential for chloride-water interactions, but continue to use $1 / r$ for intermolecular water-water interactions, as these are the conditions under which the TIP4P-FQ model was parametrized. (The $1 / r$ approximation was tested for chloride-water interactions, but was found to overstate the nearest neighbor Coulomb energies considerably.)

The Lennard-Jones interactions between TIP4P-FQ water and Drude chloride ions use the usual Lorentz-Berthelot combining rules: $\epsilon_{i j}$ is the geometric mean of $\epsilon_{i}$ and $\epsilon_{j}$, and $\sigma_{i j}$ is the additive mean of $\sigma_{i}$ and $\sigma_{j}$. The OPLS potential, on the other hand, specifies a different combining rule. This difference is not important to the properties of either model; we point it out simply to reassure the reader that we are aware of the difference and are careful to use the correct parameters for each model.

The parameters which define the properties of the Drude ion are $\alpha, \zeta, \epsilon$, and $\sigma$. We must also specify $\delta m, q$, and $\omega$, but these will have little or no effect on the properties of the model 


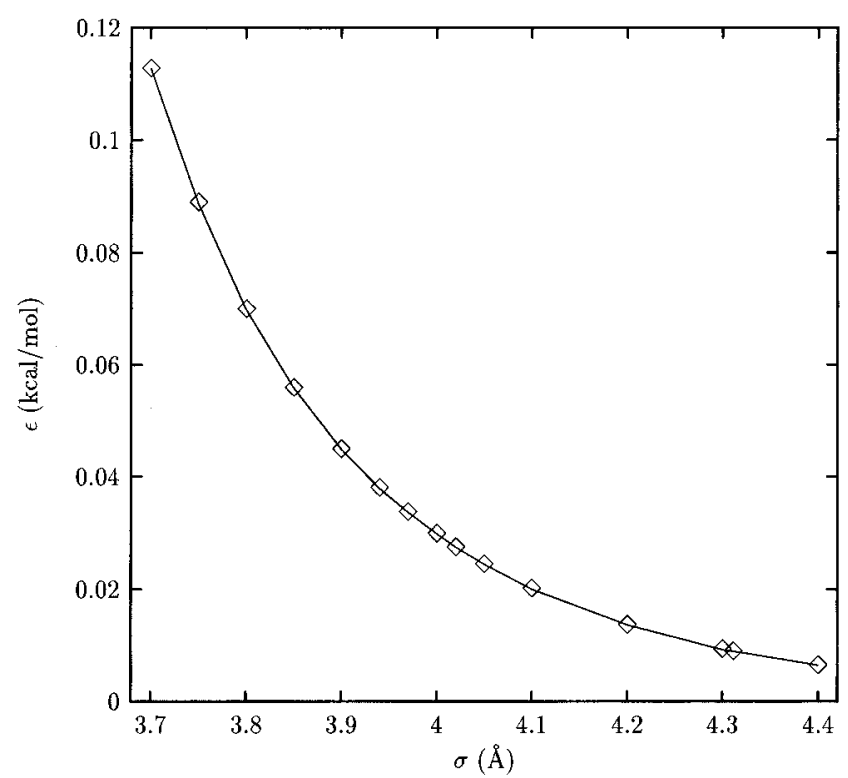

Figure 1. Lennard-Jones $(\epsilon, \sigma)$ pairs which produce reasonable $\mathrm{Cl}\left(\mathrm{H}_{2} \mathrm{O}\right)^{-}$dimer bond lengths and energies (with $\zeta=2.307 \AA^{-1}$ and $\alpha$ $\left.=3.76 \AA^{3}\right)$.

and will be chosen for computational convenience. We describe below the procedure used to parametrize the Drude ion model.

The polarizability $\alpha$ is set to $3.76 \AA^{3}$, the experimental value in aqueous solution. ${ }^{34}$ The parameter $\zeta$ specifies the delocalization of the chloride ion's charges ( $c f$. eq 4). This, together with the Lennard-Jones coefficients, controls the strength of the ion-water interactions. Thus $\zeta, \epsilon$, and $\sigma$ were varied simultaneously to reproduce the experimental values for the liquidstate $g_{\mathrm{ClO}}(r)$ peak $(3.15 \pm 0.03 \AA$, ref 35$)$ and the $298 \mathrm{~K}$ dimer enthalpy $(-15.0 \mathrm{kcal} / \mathrm{mol}$, ref 36$)$. The parameters that reproduce these values (see below) give a $\mathrm{Cl}\left(\mathrm{H}_{2} \mathrm{O}\right)^{-}$bond length of $3.04 \AA$ and energy of $-15.0 \mathrm{kcal} / \mathrm{mol}$ for the heterodimer in its minimum energy configuration; both are close to the quantum mechanical results of $3.11 \AA$ and $-14.8 \mathrm{kcal} / \mathrm{mol} .{ }^{37}$ It is perhaps worth noting that one of the most commonly quoted values for the experimental dimer enthalpy is $-13.1 \mathrm{kcal} / \mathrm{mol} .{ }^{38}$ Several existing chloride models ${ }^{28,39}$ are, unfortunately, based on this measurement, even though it is generally recognized to be erroneous. ${ }^{36,40,41}$

Using these three adjustable parameters to reproduce only two physical properties results in a manifold of potential parameter sets. Figure 1 shows the set of $(\epsilon, \sigma)$ values that produce the bond lengths and energies required above, for a value of $\zeta=2.307 \AA^{-1}$. Several points on this curve were used in 10-ps liquid simulations, and we chose the parameter set that best reproduced the experimental bulk solvation enthalpy of $-85 \mathrm{kcal} / \mathrm{mol} .^{42}$

The three remaining parameters $-\delta m, q$, and $\omega$-do not affect the physical properties of the model, except through their effects on $\alpha$ (see equation 10). For correctly integrated adiabatic dynamics (which we describe more fully in the following section), the Drude ion will have a dipole moment determined only by the instantaneous electric field of its neighbors. Thus the physical separation of the charges and the oscillations of the dipole are not relevant to the properties of the model. The charge $q$ on the lighter Drude particle was chosen to be $5|e|$ in order to maintain Drude spring displacements of $d \lesssim 0.02 \AA$, thus approximating a point dipole. The spring frequency $\omega$ was chosen to minimize any unintentional coupling with the other degrees of freedom, while still allowing the dipole to relax on a time scale faster than the nuclear motions. Certain values of $\omega$ were seen to induce resonances in the Drude spring that were related to either the fluctuating charges or the size of the time step and caused the Drude subsystem to absorb energy quite rapidly from the physical system. Values of $2 \mathrm{fs}^{-1} \leq \omega \leq 4$ $\mathrm{fs}^{-1}$ were found to be suitable, however. A frequency of $\omega=$ $3.20 \mathrm{fs}^{-1}$ was chosen for the current model, resulting in Drude subsystem heating rates of less than $0.02 \mathrm{~K}$ per picosecond of simulation. With $\alpha, q$, and $\omega$ assigned, eqs 10 and 7 dictate that $\delta m=0.090$ amu.

The full parametrization of the Drude chloride ion model is complete at this point; the parameters are summarized in Table 2.

In developing the Drude ion model, we chose to use fixed charges with different masses at the ends of a flexible spring to model the point dipole; other approaches are equally plausible. One alternative, for example, would be to use fluctuating charges on a molecular lattice. ${ }^{43-45}$ In our experience, however, closely spaced fluctuating charges can give rise to spurious charge fluctuations. Furthermore, Madden's simulations suggest that the free rotation of a spherical dipole leads to very fast thermalization with the rest of the system, ${ }^{44}$ an undesirable phenomenon in an adiabatic simulation. The current model has more in common with the shell model prevalent in simulations of ionic crystals and melts, ${ }^{33}$ which is also suitable for extended Lagrangian dynamics simulations. ${ }^{46}$

\section{Dynamics Algorithm}

3.1. Extended Lagrangian Dynamics. The fixed charge models (OPLS water, OPLS chloride) are simulated with the usual molecular dynamics techniques. Periodic boundary conditions are imposed with Ewald summation, ${ }^{47,48}$ and the velocity Verlet integrator ${ }^{49}$ is used to integrate the dynamics.

These techniques are also used for the fluctuating-charge model, but the system's Lagrangian is slightly different. Since we are treating the partial charges as additional variables, we extend the Lagrangian to include the kinetic energy of these new degrees of freedom,

$$
L=\frac{1}{2} \sum_{i \alpha} m_{i \alpha} \dot{r}_{i \alpha}^{2}+\frac{1}{2} \sum_{i \alpha} \mu_{q} \dot{q}_{i \alpha}^{2}-V[\{\mathbf{r}\},\{q\}]-\sum_{i} \lambda_{i} \sum_{\alpha \in i} q_{i \alpha}
$$

where the $m_{i \alpha}$ are the masses of the atoms and $\mu_{q}$ is a fictitious "mass" assigned to the charge degrees of freedom (which does not have the usual units of mass). The $\lambda_{i}$ are Lagrange multipliers used to maintain charge neutrality on each individual water molecule. Using this extended Lagrangian to solve for the equations of motion gives

$$
m_{i \alpha} \ddot{\mathbf{r}}_{i \alpha}=-\nabla_{\mathbf{r}_{i \alpha}} V
$$

for the physical degrees of freedom (the atom positions), and

$$
\mu_{q} \ddot{q}_{i \alpha}=-\left(\chi_{i \alpha}-\bar{\chi}_{i}\right)
$$

for the charge degrees of freedom, where

$$
\chi_{i \alpha}=\frac{\partial V}{\partial q_{i \alpha}}
$$

is the electronegativity on atom $i \alpha$ and $\bar{\chi}_{i}$ is the average electronegativity on molecule $i .^{17}$ Note that no special treatment is needed for the Drude displacement variable $\mathbf{d}$, as the motion of this coordinate is completely determined by eq 13. Similarly, the kinetic energy of the Drude displacement, (1/2) $\sum \mu \omega^{2} d^{2}$, is contained in the kinetic energy of eq 12. The usual Ewald summation and velocity Verlet algorithms can be used on this 
system with very little modification, integrating four degrees of freedom per atom instead of three.

In fluctuating-charge simulations, the charges must be kept near their minimum-energy values at all times. This corresponds to keeping the kinetic energy of the charge degrees of freedom very low, so that the charges move adiabatically on their groundstate energy surface. In practical terms, maintaining a temperature of $5 \mathrm{~K}$ or less for the charge degrees of freedom ensures that the charges remain within a few hundredths of an electron charge of their minimum-energy values and that the fictitious charge kinetic energy represents only a small perturbation on the physical Lagrangian.

The Drude ions require no special extension of the Lagrangian, since the Drude atom positions are already included in eq 12 . But the stretch of the Drude oscillator, $\mathbf{d}$, does represent a fictitious dynamic variable in the sense that it is a perturbation on the true Lagrangian. Once again, we wish this degree of freedom to remain near its minimum-energy value, oscillating about it with a very small kinetic energy. Thus we treat this degree of freedom separately in calculating the system temperature and require it to stay below $1 \mathrm{~K}$ at all times. It should be noted that if at every time step the value of $\mathbf{d}$ were calculated using energy minimization, the results would be identical to those obtained from the local field equations when using a dipole-polarizable model.

In a long simulation, both the charge and Drude degrees of freedom will eventually equilibrate thermally with the physical degrees of freedom, due to the equipartition of energy. Both models have been parametrized so as to minimize the coupling between the various subsystems, however, and the rate of heat transfer into the fictitious degrees of freedom is typically less than $0.1 \mathrm{~K} / \mathrm{ps}$.

3.2. rRESPA. The velocity Verlet integrator is used for systems containing TIP4P water, TIP4P-FQ water, and OPLS chloride ions. It could also be used to integrate systems containing Drude ions, but would have to use a much smaller time step due to the very stiff spring frequency $\omega$. Since dilute solutions of these ions will contain only a few of these fast degrees of freedom, we use the reversible reference system propagator algorithm (rRESPA) to integrate the dynamics. ${ }^{50}$

This involves subdividing the full Liouvillian,

$$
i \mathrm{~L}=\sum_{i \alpha}\left\{\mathbf{v}_{i \alpha} \cdot \nabla_{\mathbf{r}_{i \alpha}}+\frac{\mathbf{F}_{i \alpha}}{m_{i \alpha}} \cdot \nabla_{\mathbf{v}_{i \alpha}}+\dot{q}_{i \alpha} \frac{\partial}{\partial q_{i \alpha}}-\frac{\chi_{i \alpha} \partial}{\mu_{q} \partial \dot{q}_{i \alpha}}\right\}
$$

into a component that advances only the quickly oscillating Drude degrees of freedom,

$$
i \mathrm{~L}_{l}=\sum_{i \alpha \notin \text { Drude }}\left\{\mathbf{v}_{i \alpha} \cdot \nabla_{\mathbf{r}_{i \alpha}}+\frac{\mathbf{F}_{i \alpha}}{m_{i \alpha}} \cdot \nabla_{\mathbf{v}_{i \alpha}}\right\}
$$

and one which advances the remaining, slower degrees of freedom,

$i \mathrm{~L}_{h}=\sum_{i \alpha \notin \text { Drude }}\left\{\mathbf{v}_{i \alpha} \cdot \nabla_{\mathbf{r}_{i \alpha}}+\frac{\mathbf{F}_{i \alpha}}{m_{i \alpha}} \cdot \nabla_{\mathbf{v}_{i \alpha}}+\dot{q}_{i \alpha} \frac{\partial}{\partial q_{i \alpha}}-\frac{\chi_{i \alpha} \partial}{\mu_{q} \partial \dot{q}_{i \alpha}}\right\}$

A reversible integrator is then constructed by Trotter factorizing the discrete time propagator $U(\Delta t)=\exp (i \mathrm{~L} \Delta t)$,

$$
G(\Delta t)=\left[\mathrm{e}^{i L_{l} \delta t}\right]^{n_{l} / 2} \mathrm{e}^{i L_{h} \Delta t}\left[\mathrm{e}^{i L_{l} \delta t}\right]^{n_{l} / 2}
$$

where $n_{l}$ and $\delta t=\Delta t / n_{l}$ determine the accuracy at which the fast subsystem's dynamics are integrated. The simulations presented here use $n_{l}=4$, with $\Delta t=1$ fs and $\delta t=0.25$ fs.
When this rRESPA integrator is used, systems containing Drude chloride ions require only $3-30 \%$ more computer time than a comparable simulation with a fixed-charge chloride ion, despite the factor of 4 difference in time steps. (The extra cost is 3\% for a 256-molecule system without Ewald sums, 30\% for the same size system with Ewald sums: a rRESPA split of this type is considerably less efficient when Ewald sums are used.) The may be compared with an added cost of 100-300\% required by dipole-polarizable simulations. ${ }^{27}$

The rationale behind this particular rRESPA split is discussed at greater length elsewhere. ${ }^{51}$ We note here only that an alternative split, which places the heavier Drude ion charge into the slow part of the propagator, produces markedly worse energy conservation.

\section{Simulation Details}

In parametrizing and characterizing the Drude chloride ion models, several simulations were performed on bulk-phase, aqueous solutions. These periodic systems contained 255 water molecules and one chloride ion in a cubic box of side $L=$ $19.7781 \AA$, for a $\mathrm{Cl}^{-}$concentration of $0.215 \mathrm{M}$. Ewald summation was used with a screening parameter $\kappa=6.0 / L$, a real-space cutoff of $9.85 \AA \approx L / 2$, a $k$-space cutoff of $|\mathbf{n}|=5$ (257 $k$-vectors), and conducting boundary conditions. The net negative charge in the unit cell was compensated for by discarding the $\mathbf{k}=\mathbf{0}$ term in the Ewald sum; this is equivalent to applying a uniform neutralizing background charge to the unit cell. Simulations were run at $298 \pm 5 \mathrm{~K}$, starting from equilibrated configurations, in the microcanonical (constant $n V E$ ) ensemble. The fluctuating charge and/or Drude ion subsystems (if present) were quenched to their minimum-energy states when their fictitious kinetic energies exceeded 5 or $1 \mathrm{~K}$, respectively. This was generally required after 50-100 ps of dynamics.

The principal focus of this study, however, is on $\mathrm{Cl}\left(\mathrm{H}_{2} \mathrm{O}\right)_{n}{ }^{-}$ clusters. The systems studied contained between one and 255 water molecules and a single chloride ion. Periodic boundary conditions were not imposed for these clusters. When possible, these clusters were simulated at $298 \mathrm{~K}$. Some clusters with 9 $\leq n \leq 20$, however, showed a tendency to eject water molecules at this temperature and were simulated at the highest temperature for which evaporation was not observed. These cooler temperatures ranged from 240 to $280 \mathrm{~K}$. We permitted occasional evaporation events in larger clusters $(n=100$ and $n=255)$, as long as no more than $5 \%$ of the water molecules were ejected over the course of the simulation. The total simulation times (exclusive of equilibration) were at least $1 \mathrm{~ns}$ for the clusters with $n \leq 20$ and at least 300 ps for the larger clusters. The low temperatures of the adiabatic fluctuating-charge and Drude subsystems were much more easily maintained than in the bulk simulations; no quenching was required in any of the cluster simulations.

The bond constraints in the TIP4P and TIP4P-FQ models were maintained through the use of the RATTLE method. ${ }^{52} \mathrm{~A}$ time step of $\Delta t=1$ fs was used for all simulations; those involving Drude ions also used rRESPA with a small time step of $\delta t=0.25$ fs for the Drude degrees of freedom.

\section{Results}

5.1. Bulk Aqueous Solution. Although our primary concern is the structure and energetics of aqueous chloride clusters, we present the properties of the Drude ion model in bulk aqueous solution to show that it is accurate in a broad range of environments. Since ionic clusters have only a few properties that are experimentally accessible, our confidence in the 


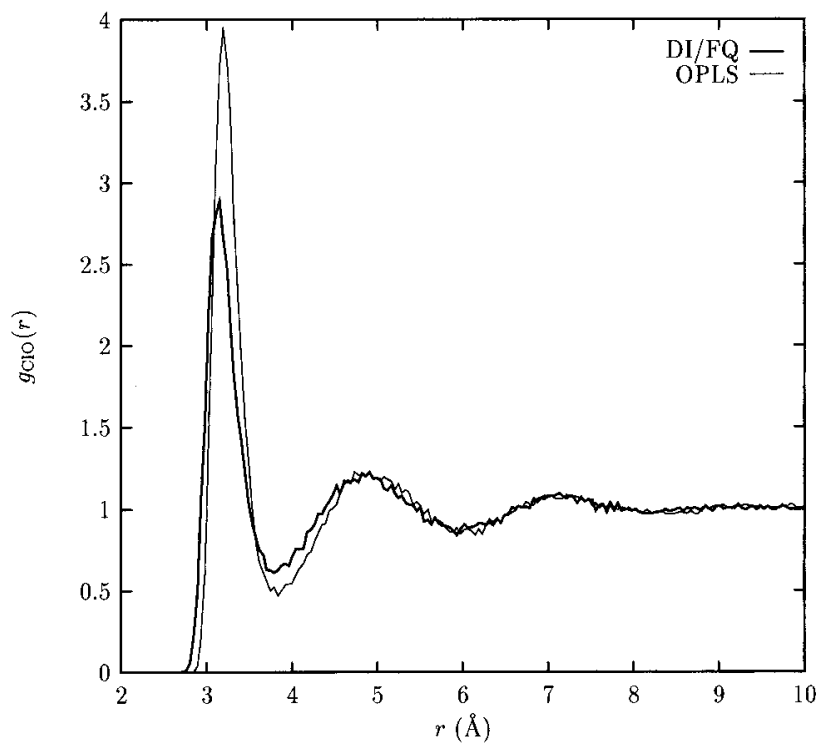

Figure 2. Chloride-oxygen pair correlation function in bulk aqueous solution for the DI/FQ (bold line) and OPLS (thin line) potentials.

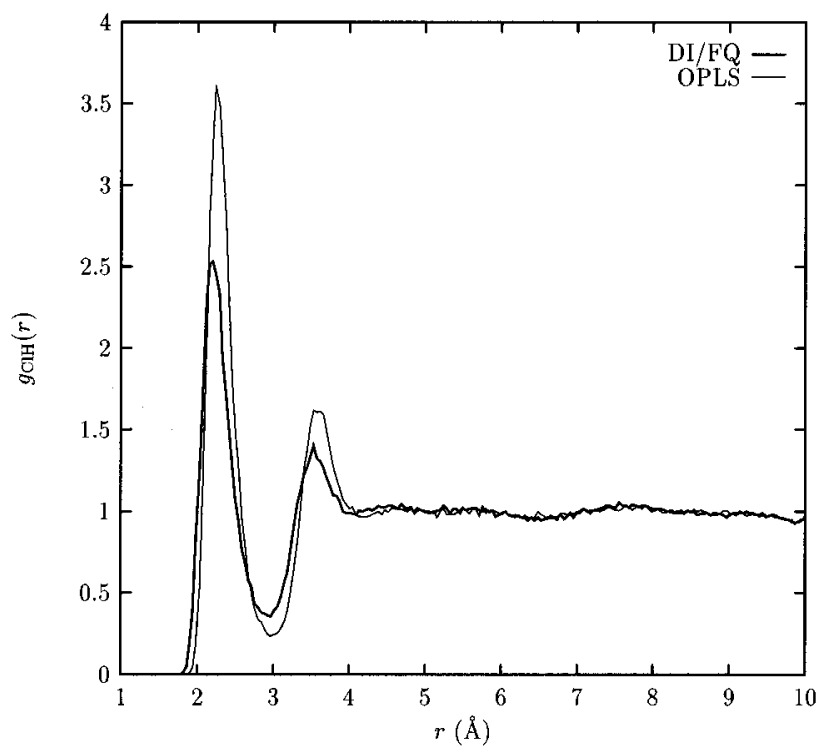

Figure 3. Chloride-hydrogen pair correlation function in bulk aqueous solution for the DI/FQ (bold line) and OPLS (thin line) potentials.

accuracy of the chloride model will depend to some extent on the model's accuracy in the bulk.

Simulations of 300 ps were performed on the Drude ion in TIP4P-FQ water (the DI/FQ system) as well as on the OPLS ion in TIP4P water (the OPLS system). The chloride-oxygen and chloride-hydrogen pair correlation functions for these simulations, $g_{\mathrm{ClO}}(r)$ and $g_{\mathrm{ClH}}(r)$, are compared in Figures 2 and 3. The positions of the peaks are similar for both models, and both reproduce the experimental peak positions quite well. ${ }^{35} \mathrm{~A}$ more difficult quantity to reproduce correctly is the coordination number $(\mathrm{CN})$ of the chloride ion, obtained by integrating the number of oxygens under the first peak of $g_{\mathrm{ClO}}(r)$. Experimental results provide a consensus estimate of 6 for the $\mathrm{CN}^{\circ} \mathrm{Cl}^{-}$, but many MD force fields give $\mathrm{CN}$ values of 7 or higher. ${ }^{35}$ The OPLS potential is one such potential, with a $\mathrm{CN}$ of 7.1. The $\mathrm{DI} / \mathrm{FQ}$ potential, on the other hand, comes quite close to experiment, with a $\mathrm{CN}$ of 6.15 .

Another property that is somewhat difficult to reproduce is the bulk solvation enthalpy, $\Delta H_{\text {solv }}$. The experimental value is estimated to be $-85.3 \mathrm{kcal} / \mathrm{mol}$, although this is to some extent dependent on simulation, since the chloride ion cannot be

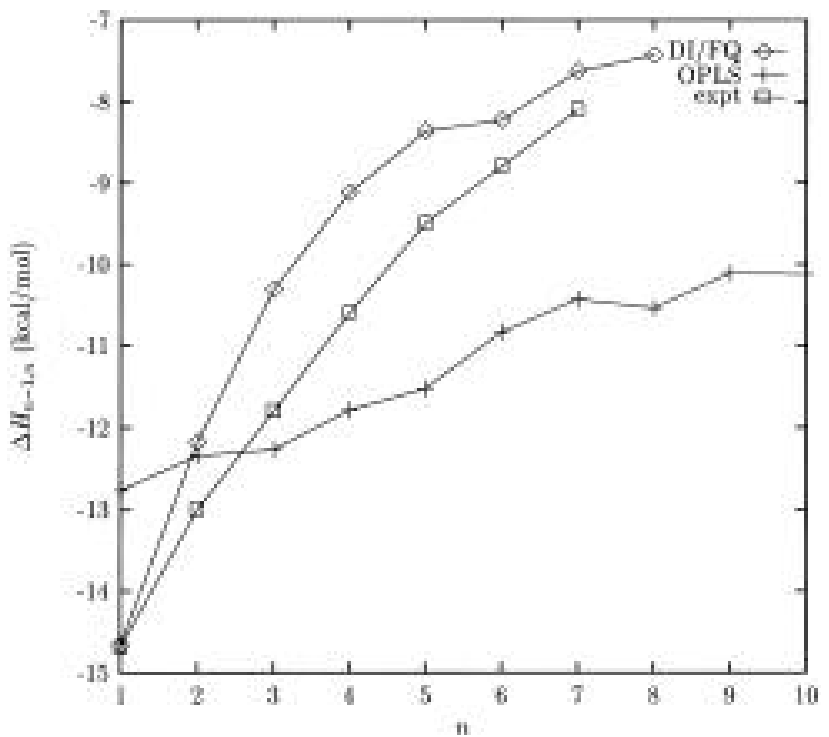

Figure 4. Clustering enthalpies for the reaction $\mathrm{Cl}\left(\mathrm{H}_{2} \mathrm{O}\right)_{n-1}{ }^{-}+\mathrm{H}_{2} \mathrm{O}$ $\rightarrow \mathrm{Cl}\left(\mathrm{H}_{2} \mathrm{O}\right)_{n}{ }^{-}$, for the DI/FQ $(\diamond)$ and OPLS (+) potentials and for experiment $(\square)$.

separated from its counterion in a physical system. ${ }^{42}$ Both the OPLS and DI/FQ potential models give an ion that is too weakly solvated, with $\Delta H_{\text {solv }}$ values of -80.2 and $-79.5 \mathrm{kcal} / \mathrm{mol}$, respectively. (Recall that this was one of the properties used in fitting the DI model; all other parameter sets from Figure 1 resulted in even more weakly solvated chlorides.)

Thus we conclude that the DI/FQ model is a slight improvement on the OPLS model in bulk solution, resulting in an equally good description of the system's energetic properties and improving on structural properties such as the coordination number. The DI/FQ model is on par with other polarizable models, such as the SPCE/POL model of Dang et al. ${ }^{23,24}$

5.2. Clusters. For the purposes of this study, though, we are more interested in $\mathrm{Cl}\left(\mathrm{H}_{2} \mathrm{O}\right)_{n}{ }^{-}$clusters. One of the few experimental quantities available for these systems is the clustering enthalpy $\Delta H_{n-1, n}$ for the association reaction

$$
\mathrm{Cl}\left(\mathrm{H}_{2} \mathrm{O}\right)_{n-1}{ }^{-}+\mathrm{H}_{2} \mathrm{O} \rightarrow \mathrm{Cl}\left(\mathrm{H}_{2} \mathrm{O}\right)_{n}{ }^{-}
$$

This represents the binding enthalpy of the $n$th water molecule to a $\mathrm{Cl}\left(\mathrm{H}_{2} \mathrm{O}\right)_{n-1}{ }^{-}$cluster and can be calculated from simulation by taking

$$
\Delta H_{n-1, n}=\bar{V}_{n}-\bar{V}_{n-1}-k T
$$

where $\bar{V}_{n}$ is the average potential energy in a simulation of a $\mathrm{Cl}\left(\mathrm{H}_{2} \mathrm{O}\right)_{n}{ }^{-}$cluster.

In Figure 4 we compare these incremental clustering enthalpies for the DI/FQ and OPLS models and experiment, ${ }^{36}$ for clusters ranging from $n=1$ to $n=7$. Simulation results are included for all clusters that could be simulated at $298 \mathrm{~K}$ without evaporation (up to $n=8$ for DI/FQ and $n=10$ for OPLS). All three curves show a decrease in binding enthalpy with increasing cluster size, as the water molecules begin to compete for the strong ion-water hydrogen bonds. The DI/FQ model reproduces the experimental curve significantly better than does the nonpolarizable OPLS model. This should not be too surprising, as the OPLS model was parametrized primarily for the liquid state. Note, however, the absence of a sharp step near $n=6$ in all of these curves. This suggests that none of the three studies shows the water molecules filling the first solvation shell completely before adding to the second shell. 


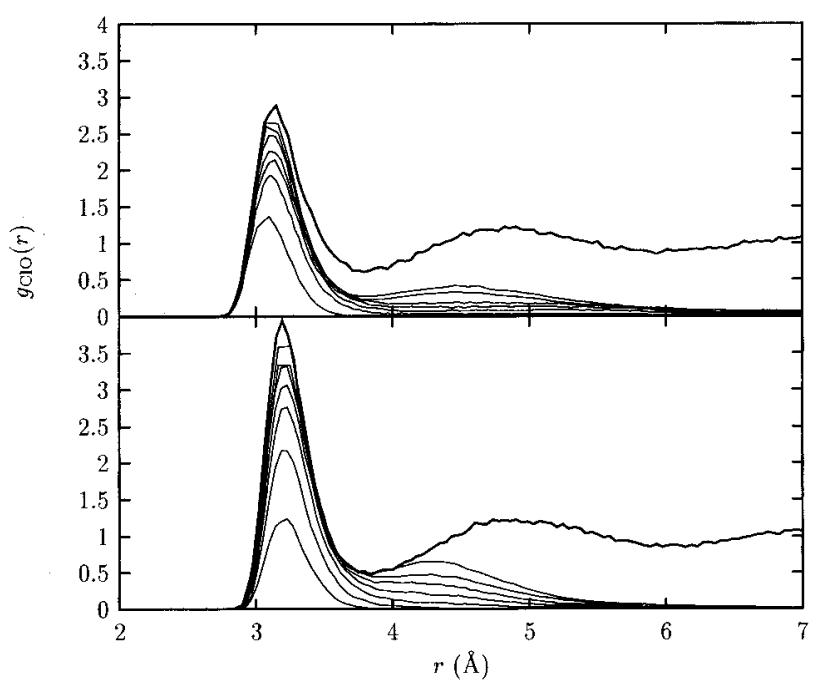

Figure 5. Chloride-oxygen pair correlation functions for $\mathrm{Cl}\left(\mathrm{H}_{2} \mathrm{O}\right)_{n}{ }^{-}$ clusters with $n=2,4,6,8,10,12$, and 14 (thin lines) and for the bulk aqueous solution (bold lines), calculated using the DI/FQ potential (upper frame) and the OPLS potential (lower frame).

Another property that is experimentally accessible for these clusters is the electrostatic stabilization energy, or the vertical excitation energy for photodetachment of an electron from the chloride ion. ${ }^{53}$ This quantity may be obtained from a simulation by calculating the energy cost associated with removing the charge on the chloride ion while freezing the nuclear coordinates but allowing the fast electronic degrees of freedom (the fluctuating charges and Drude dipole, here) to relax. The average of this energy difference over a fully equilibrated dynamics trajectory represents the stabilization energy. We have calculated this quantity for small clusters, although at temperatures significantly higher than experimental conditions. The results are in reasonable agreement with experiment, although they do not agree as well as the results of Perera and Berkowitz. ${ }^{54}$ Due to differences between the simulation and experimental conditions, however, we are not convinced that the calculated and measured photoelectron spectra are equivalent, and we do not present the results. For a rigorous comparison, the simulations would need to be performed at a much lower temperature $(\sim 70 \mathrm{~K})$, and the postejection chloride should be treated with polarizability and Lennard-Jones parameters more appropriate for the neutral species.

Since the DI/FQ model has been shown to be successful in reproducing both liquid-state properties and cluster enthalpies, it appears to be transferable over a wide range of environments. Thus we are reasonably confident that it should provide an accurate means of studying the structure of aqueous ionic clusters. By comparing the results from the DI/FQ and OPLS models, we can determine which of these properties depend on accurate treatment of the many-body polarization effects.

To begin with, we examine the $g_{\mathrm{ClO}}(r)$ correlation functions for clusters of various sizes, which should provide an indication of which solvation shells are being filled, and in what order. Some care is required in calculating a pair correlation function for a cluster system, since the simulation cell has no boundaries; the reference number density of water molecules is taken to be the same as that in the solution-phase simulations $\left(30.34 \AA^{3}\right.$ / $\mathrm{H}_{2} \mathrm{O}$ ) so that comparisons may be made between bulk and cluster systems. Figure 5 shows this $g_{\mathrm{ClO}}(r)$ function for selected cluster sizes through $n=14$ and for both the DI/FQ and OPLS models. The results are compared to the $g_{\mathrm{ClO}}(r)$ curves for the bulk aqueous solution to give an indication of where the solvation shells will eventually appear. In the DI/FQ model

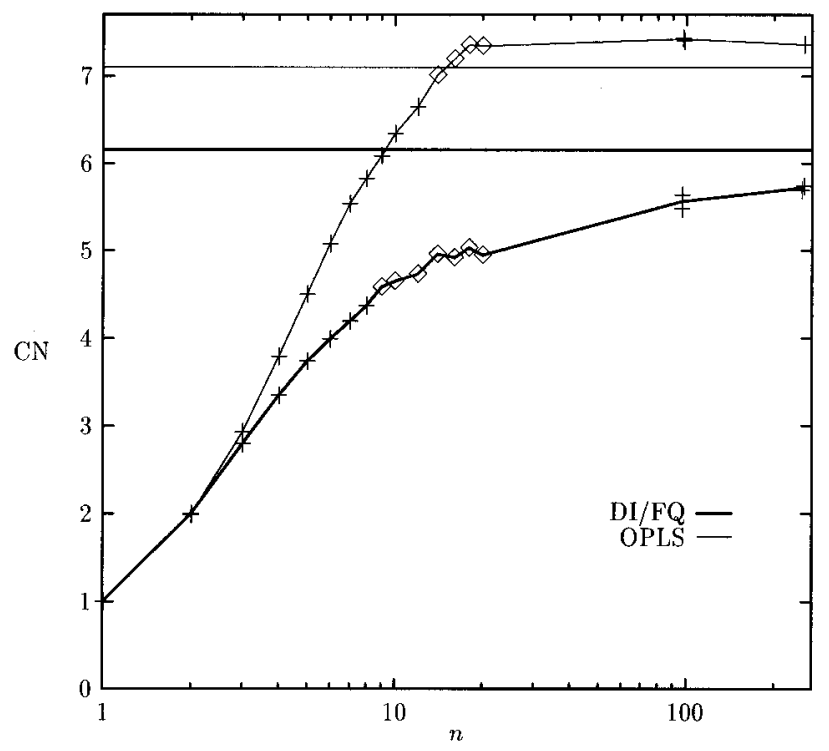

Figure 6. Chloride ion coordination number as a function of cluster size in $\mathrm{Cl}\left(\mathrm{H}_{2} \mathrm{O}\right)_{n}{ }^{-}$clusters, for the DI/FQ (bold lines) and OPLS (thin lines) potential models. Points marked with a $\diamond$ indicate clusters that were simulated at temperatures below $280 \mathrm{~K}$. The coordination numbers in bulk aqueous solution are depicted by horizontal lines.

(top frame), the water molecules begin venturing into the second shell in clusters as small as $\mathrm{Cl}\left(\mathrm{H}_{2} \mathrm{O}\right)_{4}{ }^{-}$and seem to wander quite freely about this shell. The first shell is populated slowly and incrementally and is not full by $n=14$. (Note that the secondshell structure for the $n=12$ and $n=14$ curves may be partially due to the lower temperature $(\sim 240 \mathrm{~K})$ at which these runs were conducted.) The OPLS model also begins to fill the second shell before the first is completely occupied, but in a different way. The second-shell waters do not venture as far from the chloride as in the DI/FQ model, and the first shell appears to be almost completely full by $n=14$.

To more clearly answer the question of whether the chloride ion is on the "inside" or the "outside" of the cluster, we also examine the chloride's coordination number as a function of cluster size. We calculate this by integrating the cluster's $g_{\mathrm{ClO}^{-}}$ ( $r$ ) out to the first minimum of the bulk coordination function, so that the size of the first shell is independent of the cluster size (but does depend on the potential model). If the chloride ion's first shell is fully populated (i.e. the $\mathrm{CN}$ equals or exceeds the bulk value), then we presume the chloride ion to be completely surrounded by water molecules and thus on the inside of the cluster. Conversely, if the coordination number is below the bulk value, then the ion is most likely not completely surrounded by water molecules and is at or near the surface of the cluster. (Note that this analysis ignores the possibility that the cluster density may differ substantially from the bulk density.)

Figure 6 shows this coordination number for both models as a function of cluster size. For the OPLS model, the chloride appears to become fully solvated by around $n \approx 14-18$. The DI/FQ model, on the other hand, remains only partially solvated in clusters as large as $n=255$, achieving only $93 \%$ of its full bulk coordination number. This last result was quite unexpected, particularly in light of the fact that both simulation and experiment show that chloride ions are solvated away from the surface at flat air-water interfaces. ${ }^{9,10}$ The relative solvation of the chloride ion in the DI/FQ clusters is increasing monotonically with cluster size, however, and will presumably become fully solvated for some cluster size larger than $n=255$.

Figure 7 shows representative configurations for both the DI/ FQ and OPLS Cl$\left(\mathrm{H}_{2} \mathrm{O}\right)_{100}{ }^{-}$clusters. A single configuration says 
A)

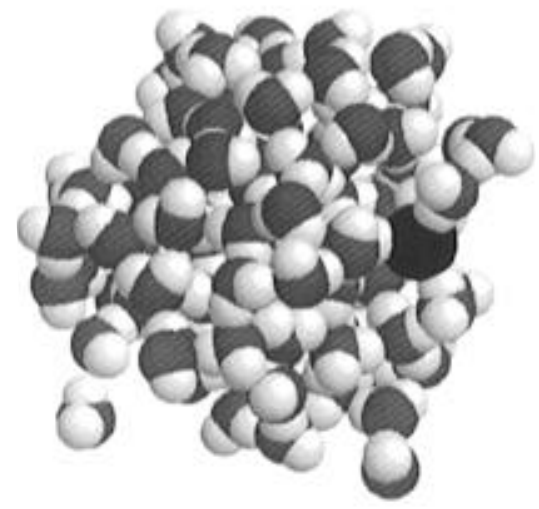

B)

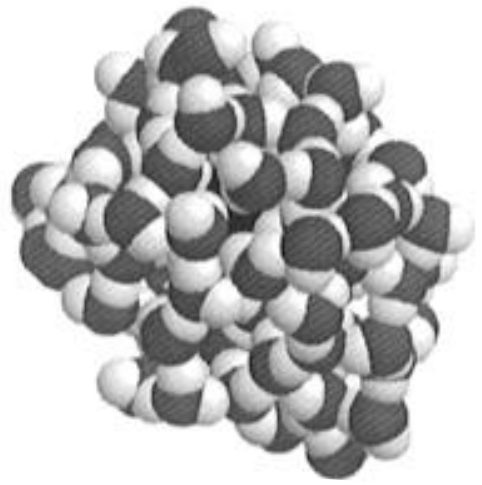

Figure 7. Typical configurations of the $\mathrm{Cl}\left(\mathrm{H}_{2} \mathrm{O}\right)_{100}{ }^{-}$clusters for (A) the DI/FQ potential model and (B) the OPLS potential model. Chloride ions are displayed in black, oxygen atoms in gray, and hydrogen atoms in white.

very little about the average position of the chloride ion, of course; Figure 7 is simply meant as an illustration of the interior $v s$ exterior solvation behavior in these clusters.

The $g_{\mathrm{ClO}}(r)$ and coordination numbers are useful, but they provide only an indirect, averaged measure of the chloride ion's position in the cluster. For a more detailed description of the distribution of chloride positions, we examine the pair correlation $g_{\mathrm{Clcom}}(r)$ between the chloride ion and the center of mass of the water molecules. (We exclude the chloride ion from the calculation of the center of mass to simplify future comparisons with other ions and to eliminate the effect that the heavy ion can have on the position of the center of mass in smaller clusters.) The similarly defined $g_{\text {Ocom }}(r)$ will also be used to describe the distribution of water molecules in the cluster.

We show these $g_{\text {Clcom }}(r)$ curves for the $n=100$ system for both potential models in Figure 8. In the 300-400 ps for which these simulations were run, the chloride could not explore the full conformational space of the cluster. Thus there is the risk that the initial position of the chloride ion could bias the results of the simulation. To avoid this complication, and as a means of determining whether the simulation was converged, we ran two simulations for each potential model: one with the chloride initially at the center of the cluster and one with the chloride initially on the surface. The $g_{\text {Clcom }}(r)$ curves for both of these runs are given in Figure 8 and demonstrate adequate (although not perfect) convergence. Also shown are the $g_{\text {Ocom }}(r)$ curves, provided primarily as an indication of the size of the cluster.

From Figure 8, it is apparent that the polarizable DI/FQ model solvates the chloride near the boundary of the cluster, while the nonpolarizable OPLS model solvates the chloride closer to the center. In the polarizable simulation, the chloride approaches

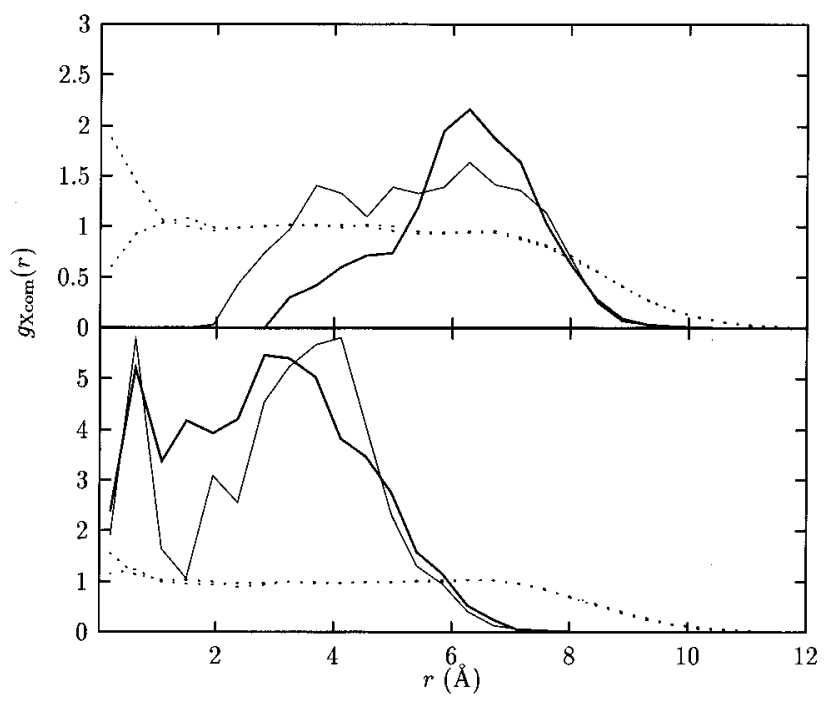

Figure 8. Distribution of the chloride ion from the center of the $\mathrm{Cl}\left(\mathrm{H}_{2} \mathrm{O}\right)_{100}{ }^{-}$cluster $\left(g_{\mathrm{Clcom}}(r)\right)$ for the DI/FQ model (top frame) and the OPLS model (bottom frame). Bold lines represent runs where the ion was initially at the center of the cluster, and thin lines represent runs where it was originally on the surface. Also shown are the distributions of water molecules $\left(g_{\text {Ocom }}(r)\right)$ for the same clusters (dotted lines), to show the total size of the cluster.

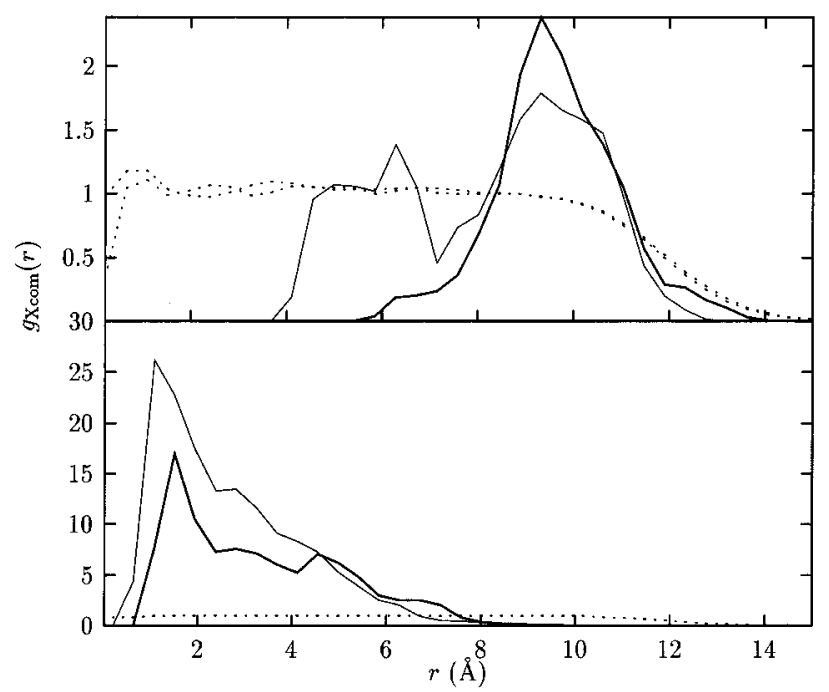

Figure 9. Distribution of the chloride ion from the center of the $\mathrm{Cl}\left(\mathrm{H}_{2} \mathrm{O}\right)_{255}{ }^{-}$cluster $\left(g_{\text {Clcom }}(r)\right)$ for the DI/FQ model (top frame) and the OPLS model (bottom frame). Bold lines represent runs where the ion was initially at the center of the cluster, and thin lines represent runs where it was originally on the surface. Also shown are the distributions of water molecules $\left(g_{\text {Ocom }}(r)\right)$ for the same clusters (dotted lines), to show the total size of the cluster.

to within $1 \AA$ or less of the cluster boundary (defined as the distance at which $\left.g_{\mathrm{Ocom}}(r)=0.5\right)$ and rarely visits the center; the nonpolarizable chloride, on the other hand, remains at least one monolayer in from the surface and spends most of its time in the core of the cluster. The situation is even more pronounced for the $\mathrm{Cl}\left(\mathrm{H}_{2} \mathrm{O}\right)_{255}{ }^{-}$cluster, as shown in Figure 9.

All of these results support the finding by Perera and Berkowitz that including many-body effects does result in different solvation of the chloride ion, with the ion located more toward the outside of the cluster in the polarizable simulations. The assertion of Jorgensen and Severance that nonpolarizable clusters also asymmetrically solvate the chloride ion primarily applies to clusters containing 18 or fewer water molecules. For larger nonpolarizable clusters the $\mathrm{Cl}^{-}$is found near the center of the cluster. 
These results can be rationalized (but not predicted) in the following way. The most important factor in determining whether an ion is solvated on the inside or the outside of a cluster is the balance between the water-water and ion-water interactions. ${ }^{5}$ If the water-water forces dominate, then the ion will not be able to form enough hydrogen bonds to make up for disturbing the hydrogen bond network of the neighboring waters. If this is the case, then the ion will move to the surface of the cluster so as to perturb the smallest possible number of hydrogen bonds. Conversely, if the ion-water bonds are strong enough, they can compensate for the structure-breaking effects they have on the nearby waters, and the ion will try to form as many bonds as it can, preferring to be solvated in the core of the cluster.

This argument also explains the tendency of the ion to withdraw from the surface as the cluster grows (given that it prefers to be there at all). For small clusters, the curvature of the surface is quite strong, and an ion located at the surface will perturb few neighboring water molecules. It is for these small clusters that the difference between surface and interior solvation is the greatest. As the cluster grows, however, the surface becomes flatter, increasing the number of water molecules whose hydrogen bond structure is broken by an ion at the surface. As this happens, the penalty for jumping into the cluster becomes smaller (relative to sitting on the surface), and the ion is more likely to make excursions into the interior of the cluster. We reiterate, however, that this is merely afterthe-fact justification, with no predictive ability. The interplay between competing forces is quite subtle and can be altered by the introduction of many-body polarization effects, as we have seen.

This naturally raises the question, what in particular is responsible for the difference in solvation behavior between the DI/FQ and OPLS models? There are several features that differ in these models: the polarization of the water molecules, the polarization of the chloride ions, and the size of the water dipole moment, to name just a few. As developers and users of potential models, we are quite interested in knowing which of these features are necessary to reproduce the asymmetric ion solvent described above and which can perhaps be dispensed with.

Perera and Berkowitz have approached this question from a somewhat different angle, concluding that the negative charge and the relatively weak electric field of the chloride ion are more important than its polarizability in driving the ion to the outside of the cluster. ${ }^{5,6}$ From the point of view of finding the simplest model that can produce these results, however, we will examine the role of polarizability in these calculations in greater detail.

To do this, we systematically remove features from the DI/ FQ model to discover which ones are indispensable.

One such modification involves removing the polarizability of the FQ water model, fixing the charges at their average values and keeping all other parameters the same. This "mean-charge" (MQ) model has been used before as a convenient way to separate the effects of explicit polarizability from the large dipole characteristic of polarizable models. ${ }^{20}$ In a simulation of a $\mathrm{Cl}\left(\mathrm{H}_{2} \mathrm{O}\right)_{100}{ }^{-}$cluster with the Drude ion and mean-charge models (DI/MQ), we find that the chloride still moves to the outside of the cluster, reaching only $93 \%$ of its bulk coordination number. This suggests that it is not the polarizability of the water molecules that drives the chloride to the outside of the cluster.

Similarly, we can also turn off the polarizability of the chloride, resulting in a Lennard-Jones chloride model (LJ) whose LJ coefficients and charge delocalization radius are reparam-
TABLE 3: Properties of Various $\mathrm{Cl}^{-}$/Water Model Combinations

\begin{tabular}{lcccc}
\hline $\begin{array}{c}\text { model } \\
\mathrm{Cl}-\text { water }\end{array}$ & $\begin{array}{c}\text { polarizable } \\
\text { water }\end{array}$ & $\begin{array}{c}\text { polarizable } \\
\mathrm{Cl}^{-}\end{array}$ & $\begin{array}{c}\text { large water } \\
\text { dipole }\end{array}$ & $\begin{array}{c}\text { surface } \\
\text { solvation }\end{array}$ \\
\hline $\mathrm{DI} / \mathrm{FQ}$ & $\sqrt{ }$ & $\sqrt{ }$ & $\sqrt{ }$ & $\sqrt{ }$ \\
$\mathrm{LJ} / \mathrm{FQ}$ & $\sqrt{ }$ & $\sqrt{ }$ & $\sqrt{ }$ & $\sqrt{ }$ \\
$\mathrm{DI} / \mathrm{MQ}$ & & & $\sqrt{ }$ & $\sqrt{ }$ \\
LJ/MQ & & $\sqrt{ }$ & & \\
DI/OPLS & & & & \\
LJ/OPLS & & & &
\end{tabular}

etrized using the same procedure described earlier for the DI model. (This results in values of $\epsilon=0.0358 \mathrm{kcal} / \mathrm{mol}, \sigma=$ $4.00 \AA$, and $\zeta=2.415 \AA^{-1}$.) This model can then be used with either the TIP4P-FQ or MQ models; both combinations again result in a chloride ion near the surface of the $n=100$ and $n=255$ clusters (relative solvation of 90-92\%).

As a further test, we again reparametrized the Drude ion model, this time against the nonpolarizable TIP4P water model, and without using delocalized charges (obtaining $\epsilon=0.7957$ $\mathrm{kcal} / \mathrm{mol}, \sigma=3.74 \AA$, with $\alpha, q, \delta m$, and $\omega$ unchanged). This combination resulted in full interior solvation of the chloride ion (relative solvation of 104\%). These last two results suggest that the chloride polarizability also does not appear to be the determining factor in whether the chloride moves to the surface.

So, how are we to interpret these simulations? It may help to consider Table 3, in which we compare the features of the various hybrid models tested. We compare them on the basis of whether the water and chloride ion were polarizable, as well as whether the water model had a large or a small average dipole moment. The FQ and MQ models are classified as having a large dipole $(\langle\mu\rangle=2.62 \mathrm{D})$, while TIP4P has a small dipole $(\mu$ $=2.18 \mathrm{D})$. The property that correlates best with the asymmetric solvation of the chloride ion is the strength of the water dipole.

A large dipole moment is known to be important in reproducing liquid-state water properties, such as the static dielectric constant. ${ }^{16}$ Fixed-charge models with dipoles this strong tend to be too strongly bound, however, and their dynamic properties too slow. ${ }^{17,55}$ Polarizable models, on the other hand, can maintain a strong average dipole while allowing fluctuations to decrease that dipole when necessary to diffuse or rotate away from an unfavorable conformation. This combination of features results in an accurate representation of both the dielectric and dynamic properties of liquid water.

The case with clusters appears to be analogous. The polarizability of the water model is not strictly necessary if we care only about solvating the chloride ion at the surface of the cluster; a large water dipole seems sufficient for that purpose. Larger dipoles strengthen the hydrogen bond network, thereby excluding the ion from the interior of the cluster so that it breaks fewer hydrogen bonds. Yet the nonpolarizable models such as the DI/MQ model explored above suffer in other ways, with water diffusion rates that are too slow and cluster enthalpies that are too tightly bound. For a water model to solvate chloride ions with the correct structure and energetics, it must be polarizable, as well as strongly polar.

\section{Discussion}

We began this work with the goal of answering several questions about the solvation of chloride ions in water clusters. These have now been answered.

We find, first of all, that there are important differences in the structure of $\mathrm{Cl}\left(\mathrm{H}_{2} \mathrm{O}\right)_{n}{ }^{-}$clusters when they are simulated with and without explicit polarizability. Specifically, the polarizable model used here is more accurate in its estimation of the binding 
enthalpies of water molecules to these clusters and predicts that the chloride ion is solvated near the outside of the cluster. Surprisingly, this surface solvation persists for cluster sizes as large as $n=100$ and $n=255$, although the ion becomes more fully solvated as the cluster size increases.

These results are different from those obtained with a nonpolarizable model, which predicts interior solvation for clusters with $n=18$ or more. The reason for this difference appears to be primarily the stronger dipole moment in the polarizable water model; the polarizability acts to moderate the effects of the larger dipole, permitting fluctuations in the dipole strength and allowing the water molecules to remain mobile. Chloride ion polarizability appears to be unnecessary in observing these effects.

One question remains unanswered: what is the threshold cluster size beyond which the ion will move into the interior of a polarizable water cluster? Although Figure 6 appears to indicate that the ion will become fully solvated at some point beyond $n=255$, we must consider the possibility that the FQ model will only partially solvate the ion at a flat interface. We are currently investigating this interfacial system to ensure that this is not the case.

Acknowledgment. This work was supported by the NSF (CHE-91-22506) and the Maui High Performance Computing Center.

\section{References and Notes}

(1) Castleman, A. W.; Keesee, R. G. Chem. Rev. 1986, 86, 589.

(2) Jorgensen, W. L.; Severance, D. L. J. Chem. Phys. 1993, 99, 4233.

(3) Perera, L.; Berkowitz, M. L. J. Chem. Phys. 1991, 95, 1954.

(4) Perera, L.; Berkowitz, M. L. J. Chem. Phys. 1993, 99, 4236.

(5) Perera, L.; Berkowitz, M. L. J. Chem. Phys. 1992, 96, 8288.

(6) Perera, L.; Berkowitz, M. J. Chem. Phys. 1994, 100, 3085.

(7) Gowda, B. T.; Benson, S. W. J. Comp. Chem. 1983, 4, 283.

(8) Rips, I.; Jortner, J. J. Chem. Phys. 1992, 97, 536.

(9) Randles, J. E. B. Phys. Chem. Liq. 1977, 7, 107.

(10) Wilson, M. A.; Pohorille, A. J. Chem. Phys. 1991, 95, 6005.

(11) Jorgensen, W. L.; Chandresekhar, J.; Madura, J. D.; Impey, R. W.; Klein, M. L. J. Chem. Phys. 1983, 79, 926.

(12) Shepard, A. C.; Beers, Y.; Klein, G. P.; Rothman, L. S. J. Chem. Phys. 1973, 59, 2254.

(13) Coulson, C. A.; Eisenberg, D. Proc. R. Soc. London Ser. A 1966, $291,445$.

(14) Barnes, P.; Finney, J. L.; Nicholas, J. D.; Quinn, J. E. Nature 1979, $282,459$.

(15) Carnie, S. O.; Patey, G. N. Mol. Phys. 1982, 47, 1129.

(16) Sprik, M. J. Chem. Phys. 1991, 95, 6762. 6141.
(18) Rick, S. W.; Stuart, S. J.; Bader, J. S.; Berne, B. J. J. Mol. Liq. 1995, 65/66, 31 .

(19) Rick, S. W.; Berne, B. J. J. Am. Chem. Soc. 1996, 118, 672.

(20) Bader, J. S.; Berne, B. J. J. Chem. Phys. 1996, 104, 1293.

(21) Andersen, H. C. J. Chem. Phys. 1980, 72, 2384.

(22) Car, R.; Parrinello, M. Phys. Rev. Lett. 1985, 55, 2471.

(23) Caldwell, J.; Dang, L. X.; Kollman, P. A. J. Am. Chem. Soc. 1990, 112,9144 .

(24) Dang, L. X.; Rice, J. E.; Caldwell, J.; Kollman, P. A. J. Am. Chem. Soc. 1991, 113, 2481 .

(25) Parr, R. G.; Donelly, R. A.; Levy, M.; Palke, W. E. J. Chem. Phys. 1978, 68, 3801.

(26) Parr, R. G.; Pearson, R. G. J. Am. Chem. Soc. 1983, 105, 7512.

(27) Ahlstroöm, P.; Wallqvist, A.; Engström, S.; Jönsson, B. Mol. Phys. 1989, 68, 563.

(28) Chandrasekhar, J.; Spellmeyer, D. C.; Jorgensen, W. L. J. Am. Chem. Soc. 1984, 106, 903.

(29) Stuart, S. J. Ph.D. Thesis, Columbia University, New York, NY, 1995.

(30) Hoye, J.; Stell, G. J. Chem. Phys. 1980, 73, 461.

(31) Pratt, L. R. Mol. Phys. 1980, 40, 347.

(32) Cao, J.; Berne, B. J. J. Chem. Phys. 1993, 99, 6998.

(33) Sangster, M. J. L.; Dixon, M. Adv. Phys. 1976, 23, 247.

(34) Pyper, N. C.; Pike, C. G.; Edwards, P. P. Mol. Phys. 1992, 76, 353.

(35) Ohtaki, H.; Radnai, T. Chem. Rev. 1993, 93, 1157.

(36) Hiraoka, K.; Mizuse, S. Chem. Phys. 1987, 118, 457.

(37) Zhao, X. G.; Gonzalez-Lafont, A.; Truhlar, D. G.; Steckler, R. J. Chem. Phys. 1991, 94, 5544.

(38) Arshadi, M.; Yamdagni, R.; Kebarle, P. J. Phys. Chem. 1970, 74, 1475 .

(39) Smith, D. E.; Dang, L. X. J. Chem. Phys. 1994, 100, 3757. 3308 .

(41) Keesee, R. G.; Castleman, A. W., Jr. Chem. Phys. Lett. 1980, 74, 139.

(42) Rashin, A. A.; Honig, B. J. Phys. Chem. 1985, 89, 5588.

(43) Sprik, M.; Klein, M. L. J. Chem. Phys. 1988, 89, 7556.

(44) Wilson, M.; Madden, P. A. J. Phys.: Condens. Matter 1993, 5, 2687.

(45) Dinur, U. J. Phys. Chem. 1993, 97, 7894.

(46) Mitchell, P. J.; Fincham, D. J. Phys.: Condens. Matter 1993, 5 , 1031.

(47) Ewald, P. Ann. Phys. 1921, 64, 253.

(48) Berthaut, F. J. Phys. Radium 1952, 13, 499.

(49) Swope, W. C.; Andersen, H. C.; Berens, P. H.; Wilson, K. R. J. Chem. Phys. 1982, 76, 637.

(50) Tuckerman, M.; Berne, B. J.; Martyna, G. J. J. Chem. Phys. 1992, $97,1990$.

(51) Stuart, S. J.; Zhou, R.; Berne, B. J. J. Chem. Phys., in press.

(52) Andersen, H. C. J. Comput. Phys. 1983, 52, 24.

(53) Markovich, G.; Pollack, S.; Giniger, R.; Cheshnovsky, O. J. Chem. Phys. 1994, 101, 9344.

(54) Perera, L.; Berkowitz, M. L. J. Chem. Phys. 1993, 99, 4222.

(55) Sprik, M. J. Phys. Chem. 1991, 95, 2283.

JP961076D 